STUDI

FRANCESI

\section{Studi Francesi}

Rivista quadrimestrale fondata da Franco Simone

165 (LV | III) | 2011

LA RAPPRESENTAZIONE DELLA MADRE NELLA

LETTERATURA FRANCESE DEL NOVECENTO a cura di Dario Cecchetti e Michele Mastroianni

\title{
Kathleen Gyssels, Passes et impasses dans le comparatisme postcolonial caribéen. Cinq traverses
}

\section{Carminella Biondi}

\section{OpenEdition \\ Journals}

\section{Édition électronique}

URL : http://journals.openedition.org/studifrancesi/5177

DOI : 10.4000/studifrancesi.5177

ISSN : 2421-5856

\section{Éditeur}

Rosenberg \& Sellier

\section{Édition imprimée}

Date de publication : 1 décembre 2011

Pagination : 690-692

ISSN : 0039-2944

\section{Référence électronique}

Carminella Biondi, «Kathleen Gyssels, Passes et impasses dans le comparatisme postcolonial caribéen. Cinq traverses », Studi Francesi [En ligne], 165 (LV | III) | 2011, mis en ligne le 30 novembre 2015, consulté le 08 janvier 2021. URL : http://journals.openedition.org/studifrancesi/5177 ; DOI : https:// doi.org/ERREUR PDO dans /localdata/www-bin/Core/Core/Db/Db.class.php L.34 : SQLSTATE[HY000] [2006] MySQL server has gone away

Ce document a été généré automatiquement le 8 janvier 2021.

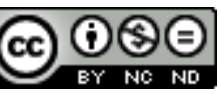

Studi Francesi è distribuita con Licenza Creative Commons Attribuzione - Non commerciale - Non opere derivate 4.0 Internazionale. 


\title{
Kathleen Gyssels, Passes et impasses dans le comparatisme postcolonial caribéen. Cinq traverses
}

\author{
Carminella Biondi
}

\section{RÉFÉRENCE}

KATHLEEN GYSSELS, Passes et impasses dans le comparatisme postcolonial caribéen. Cinq traverses, Paris, Champion, 2010, «Bibliothèque de littérature générale et comparée», pp. 432.

1 L'introduction, où l'auteur précise, comme il se doit, l'objet et les finalités de son essai, est précédée d'une page réunissant quelques citations de critiques qui soulignent la nécessité pour les études postcoloniales d'être comparatistes et le fait qu'il s'agit pour le moment d'une "promesse qui n'a pas été réalisée». Une ouverture qui en dit déjà long sur les intentions de l'auteur. Les cinq traverses du sous-titre se référant au comparatisme postcolonial caribéen concernent cinq auteurs des Antilles françaises comparés à cinq auteurs américains (même si Danticat est d'origine haïtienne) de langues anglaises, qui n'appartiennent pas tous à l'aire de la Caraïbe. Il s'agit de Maryse Condé, Léon Damas, Dany Laferrière, Jean-Claude Fignolé et Édouard Glissant qui dialoguent respectivement avec Toni Morrison, James Baldwin, Edwige Danticat, Madison Smart-Bell et Wilson Harris.

Dans l'introduction, Kathleen Gyssels insiste à juste titre sur le fait que la "pensée archipélique», sauf quelques exceptions, a plus diffracté qu'elle n'a réuni, et que la différence culturelle et linguistique entre les îles de la Caraïbe, nonobstant l'histoire et la géographie communes, a produit plus d'impasses que de passes. Une situation de claustration qui ne semble pas sur le point d'être démêlée: «La méfiance et la méconnaissance réciproques perdureront aussi longtemps que les uns et les autres privilégieront leur propre discipline, leur propre appareil conceptuel, maintenant 
fermes les cloisonnements qui, pour un domaine d'investigation comme celui de la Caraïbe, sont néfastes» (p. 17). Une autre limite est à rechercher dans le choix, peutêtre inévitable mais délétère, surtout de la part des écrivains affirmés, de s'adresser aux capitales de la langue de référence pour publier leurs œuvres, ce qui empêche la naissance et l'affirmation de maisons d'édition dans l'archipel.

Kathleen Gyssels se propose de commencer à rompre ce monadisme en établissant un rapport entre écrivains de langue française et de langue anglaise sur la base d'éléments biographiques, thématiques, stylistiques communs ou proches. Il pourrait paraître étrange que le choix, pour les écrivains de langue anglaise, ne concerne pas d'auteurs caribéens, exception faite pour Danticat, Haïtienne, et pour Wilson Harris, Guyanais, mais les résultats sont malgré tout appréciables et contribuent à ouvrir des perspectives. Ils mettent non seulement en évidence les avantages herméneutiques du comparatisme, mais aussi les apories de son manque. Sur le plan théorique et sur la fonction de rupture salutaire que cet essai représente dans le domaine des études postcoloniales en général et caribéennes en particulier, le jugement ne peut donc être que très positif. Sur la nature et les résultats des rapports établis entre les deux fronts linguistiques, on peut soulever des réserves qui ne concernent pas la méthode, mais une différente appréciation des écrivains étudiés, ce qui entre, évidemment, dans le domaine des goûts personnels, même si on a parfois l'impression que les jugements négatifs sur un auteur sont un peu trop marqués, comme nous le verrons dans l'analyse des chapitres.

4 Le premier, intitulé «Les Médées noires dans Jazz et Célanire coup-coupé» (pp. 31-91), établit un rapport entre Toni Morrison et Maryse Condé, focalisé sur les deux romans indiqués dans le titre, même si cette comparaison ne peut ignorer les deux romans que les écrivaines ont consacrés à la narration de deux procès presque occultés de matricide (Beloved, 1987 et Moi, Tituba sorcière, 1986), donc à deux «métafictions historiographiques» qui visent au rachat de la femme noire: «La comparaison entre les romans de Morrison et de Condé a révélé combien l'historiographie métafictive consistait à sortir de l'ombre la femme noire meurtrière à force d'être meurtrie» (p. 88). Tout en visant un même but et avec des parcours narratifs très proches (selon Gyssels, Morrison et Condé écrivent toujours le même roman), les résultats sont toutefois bien différents car, contrairement au prix Nobel, Condé «se complaît dans le "middlebrow postcolonial"» (p. 89), dans une littérature light, tandis que Morrison, elle, reste dans le domaine du «highbrow postcolonial». Le chapitre est convaincant et, grâce au jeu de miroirs qu'il établit, il contribue non seulement à une meilleure connaissance des deux écrivaines, mais aussi à la mise en place des premiers stades d'un discours critique qui viserait à lire les faits littéraires dans leur dimension globale. Comme tous ceux qui vont suivre, il est aussi appréciable par la richesse des références, critiques et littéraires, qui révèle une connaissance peu commune de la littérature primaire et secondaire de la Caraïbe.

5 Le deuxième chapitre est consacré à Damas et à Baldwin: «Le gay savoir de Léon Damas et de James Baldwin. Relire Black-Label et Giovanni's Room» (pp. 93-155). Gyssels affirme très clairement que Damas n'était pas homosexuel (p.146), mais que son expérience existentielle et une certaine attitude d'esprit le rapprochent de Baldwin et de son écriture: «Écritures de désillusion et de déception amoureuses, Black-Label et Giovanni's Room travestirent le tabou de la corporalité et de la sexualité noires, de l'amour interracial ainsi que celui de nature homosexuelle. À travers des images corporelles 
(limogeage) et spatiales (confinement, emprisonnement) s'expriment les troubles d'une virilité irréconciliable avec les "attentes" des Autres, c'est-à-dire les Blancs» (p. 154). De cette confrontation résultent deux portraits et deux écritures qui, s'éclairant réciproquement, aident à mieux situer les deux écrivains dans le contexte plus général de «l'écriture noire» de l'après-guerre et de l'écriture postcoloniale. La comparaison fait émerger des nuances de l'œuvre damassienne qui ne sont pas faciles à saisir et permet à Gyssels d'affirmer, à juste titre, que Damas «n'a jamais été apprécié à sa juste valeur» (p. 155). Elle arrive même à soutenir que, dans Latitudes, il «devança une "Littérature-monde"».

6 Le troisième chapitre met en parallèle deux écrivains de la diaspora haïtienne: "Comment lire Laferrière sans se fatiguer? Laferrière vs Danticat». Le choix du titre cette fois n'est pas neutre et indique très clairement quel est l'auteur sur lequel se focalise l'attention de la critique et quel sera le ton de l'approche. Ce déséquilibre dénoncé par le titre et confirmé dans le texte produit une comparaison peu convaincante et surtout improductive sur le plan critique, car ce qui est dit à propos de chacun de deux écrivains pouvait se dire sans besoin de rapprochement: ils ne s'éclairent pas réciproquement. En extrême synthèse, pour Gyssels, Laferrière est un écrivain qui a tout joué sur la provocation et sur l'apparence, sans jamais sortir de la banalité, du stéréotype et souvent de l'imitation: un petit égoïste qui a tant voyagé et n'a su rien voir autour de lui, ni hommes, ni lieux, qui n'a jamais été proche de son peuple, un écrivain blagueur, sans programme, qui a inventé «une machinerie éhonté» (p. 221), etc., contrairement à Edwidge Danticat, qui, elle, s'est ancrée à New York, mais qui est toujours restée à l'écoute de la vie et du drame d'Haïti, évitant toute exposition médiatique. Or, Dany Laferrière a sans doute cherché la provocation dans ses premiers romans, il aime à apparaître et il a peut-être tant d'autres défauts; il se peut aussi qu'il soit surestimé par la critique, mais le qualifier de «nul» me paraît excessif. Un critique est évidemment libre d'exprimer ses prédilections et ses aversions, mais une déclaration sans nuances de nullité d'un auteur rend difficile et peu féconde toute discussion.

7 Le chapitre suivant, intitulé «L'Haïtienne et la Révolution, revues par Madison SmartBell et Jean-Claude Fignolé» (pp. 224-293), se penche sur deux réécritures de la révolte noire d'Haïti et en particulier sur deux représentations de son "héros», Toussaint Louverture. Smart-Bell publie entre 1994 et 2004 une trilogie sur la révolte noire et le personnage de Toussaint: All Souls' Rising, traduit en français sous le titre Le soulèvement des âmes (1996), suivi de Master of the crossroad (2000), traduit en 2004 avec le titre Le maitre des carrefours, et de The Stone that the builder refused (2004), traduit en 2007: La pierre $d u$ bâtisseur. Une trilogie romanesque imposante qui fait renaitre un moment crucial de l'histoire d'un peuple et de la vie d'un homme qui s'est chargé de cette histoire. Son ouvrage est comparé à celui de l'écrivain haïtien Jean-Claude Fignolé qui brosse, lui aussi, une histoire du héros haïtien par excellence, tout en soulignant, dès le titre, l'intrusion «irrespectueuse» de l'auteur dans cette histoire: Moi, Toussaint Louverture... avec la plume complice de l'auteur (2004). D'autres textes de Fignolé, aussi bien que ceux d'autres écrivains qui se sont intéressés à la vie de Toussaint, sont convoqués au cours de cette comparaison, qui reste toutefois focalisée sur les textes indiqués. 1994, bicentenaire de la première abolition de l'esclavage noir de la part de la France, et 2004, bicentenaire de la proclamation de l'indépendance d'Haïti sont des anniversaires importants qui auraient fait penser à une réécriture tendant à mythifier les événements, tandis que, comme l'écrit Gyssels, «Contre toute attente, les deux 
auteurs démystifient l'aura dans laquelle baigne Toussaint Louverture et sortent des coulisses de l'Histoire quelques acteurs moins connus» (p. 229). Ils s'opposent à un regard univoque sur les événements et en proposent une «résurrection» problématique, même si le regard spiraliste de l'écrivain haïtien, plus impliqué dans les misères du présent, est plus sévère que celui de l'écrivain «encyclopédique» étasunien. C'est un chapitre important qui, grâce à la lecture spéculaire de deux auteurs engagés dans la réécriture d'un moment crucial de l'histoire des Noirs américains, contribue à une meilleure connaissance des faits (même s'il s'agit d'ouvrages de fiction), des deux auteurs et de l'aire de la Caraïbe.

8 Le cinquième et dernier chapitre met en relation deux écrivains majeurs de la Caraïbe qui, selon Gyssels, «jouissent d'un renom toutefois disproportionné» (p. 299). Le chapitre est intitulé: "Wilson Harris et Édouard Glissant: deux Isolés soleilsI» (pp. 294-349). Le sous-titre renvoie au roman Isolé soleil (1981) de l'écrivain guadeloupéen Daniel Maximin, que Gyssels évoque en ouverture de chapitre: «Dans Les Fruits du cyclone. Une géopoétique de la Caraibe, Daniel Maximin tente de combler le fossé qui sépare les voix anglo-caribéennes des voix franco-caribéennes...» (p. 295). La critique s'engage dans le même chemin avec des résultats très positifs. Les ressemblances qu'elle détecte entre l'écrivain guyanais et l'écrivain martiniquais sont impressionnantes: «Harris comme Glissant évoquent [sic] maintes fois le vertige, le tourbillon, la spirale qui les habite [...]. D'où un récit audacieusement incohérent, émis par un déparleur qui comme le sujet envoûté ou le possédé chamanique parle toutefois vrai [...]. Déménageant les langues, ils transbahutent, ils tombent dans la folie du monde, ils sont les prophètes de la Relation, conclut le Martiniquais comme s'il parlait de son Divers» (p. 349). C'est le chapitre le plus stimulant du livre, celui qui, mieux que tous les autres, prône la nécessité d'un comparatisme caribéen. En réalité tout le volume, malgré les quelques réserves avancées (qui, d'ailleurs, offrent elles aussi matière à la discussion) est à considérer comme un texte de référence pour les études caribéennes et comme une contribution importante aux recherches dans le domaine du comparatisme postcolonial. 